This is the peer reviewed version of the following article:

Tyteca, E., De Vos, J., Vankova, N., Cesla, P., Desmet, G. and Eeltink, S. (2016), Applicability of linear and nonlinear retention-time models for reversed-phase liquid chromatography separations of small molecules, peptides, and intact proteins. J. Sep. Science, 39: 1249-1257. doi:10.1002/jssc.201501395

This article may be used for non-commercial purposes in accordance With Wiley-VCH Terms and Conditions for self-archiving".

This postprint version is available from $\underline{\text { http://hdl.handle.net/10195/67569 }}$ 


\title{
Applicability of linear and nonlinear retention-time models for reversed-phase liquid chromatography separations of small molecules, peptides, and intact proteins
}

\author{
Eva Tyteca ${ }^{1}$ Jelle De Vos ${ }^{1}$ Nikola Vankova ${ }^{1,2}$ Petr Cesla $^{2}$ Gert Desmet ${ }^{1}$ Sebastiaan Eeltink $^{1}$ \\ ${ }_{1}^{1}$ Department of Chemical Engineering, Vrije Universiteit Brussel, Brussels, Belgium \\ ${ }^{2}$ Faculty of Chemical Technology, Department of Analytical \\ Chemistry, University of Pardubice, Pardubice, Czech Republic
}

\begin{abstract}
The applicability and predictive properties of the linear solvent strength model and two non- linear retention-time models, i.e., the quadratic model and the Neue model, were assessed for the separation of small molecules (phenol derivatives), peptides, and intact proteins. Retention-time measurements were conducted in isocratic mode and gradient mode ap- plying different gradient times and elution-strength combinations. The quadratic model provided the most accurate retention-factor predictions for small molecules (average abso- lute prediction error of 1.5\%) and peptides separations (with a prediction error of 2.3\%). An advantage of the Neue model is that it can provide accurate predictions based on only three gradient scouting runs, making tedious isocratic retention-time measurements obsolete. For peptides, the use of gradient scouting runs in combination with the Neue model resulted in better prediction errors $(<2.2 \%)$ compared to the use of isocratic runs. The applicability of the quadratic model is limited due to a complex combination of error and exponential functions. For protein separations, only a small elution window could be applied, which is due to the strong effect of the content of organic modifier on retention. Hence, the linear retentiontime behavior of intact proteins is well described by the linear solvent strength model. Prediction errors using gradient scouting runs were significantly lower (2.2\%) than when using isocratic scouting runs (3.2\%).
\end{abstract}

Keywords: Linear solvent strength model / Method development / Neue-Kuss model / Retention-time prediction / Selectivity

\section{Introduction}

It is generally accepted that the retention of small nonpolar molecules analyzed in RP-LC mode is dominated by partitioning [1]. When operating the column in isocratic mode, baseline separation of a mixture with components that have a wide range in hydrophobicity is often not achievable within a reasonable time. To overcome the "general-elution problem," a gradient in solvent strength can be applied [2]. Because the peak width in gradient mode is mainly determined by the retention factor at the time of elution, a gradient separation results in narrow and approximately constant peak widths, and hence, comparable detection sensitivity.

The development of retention models, describing the retention factor $(k)$ as function of mobile-phase composition $(<!)$ is crucial for method development strategies, in which the

Correspondence: Dr. Sebastiaan Eeltink, Department of Chemical Engineering, Vrije Universiteit Brussel, Pleinlaan 2, B-1050, Brussels, Belgium

E-mail: seeltink@vub.ac.be

Abbreviations: ACN, acetonitrile; FA, formic acid; LSS, linear solvent strength; MW, molecular weight aim is to achieve the desired separation performance within the shortest possible analysis time. Method development for the analysis of mixtures usually requires a great deal of expertise and can be time consuming (up to several weeks of work), because of the high probability of overlapping peaks $[3,4]$ and the sensitive dependency of the retention time of the individual analytes on, for example, the employed gradient slope. Since the late 1970's many attempts have been made to develop retention-time models and implement them in computer-assisted method development (CAMD) software, such as Chromsword [5] and DryLab [6]. Different retentiontime models in RP-LC have been developed with applicable expressions describing the relation between $k$ and $<$ ! , such as the linear solvent strength (LSS) model [7], the quadratic retention-time model [8-10], and the Neue model [11]. The LSS model introduced by Snyder and Dolan in the 1980's is most frequently applied in method development strategies, and describes isocratic retention in RP-LC, which is expressed as [7]:

$\ln (k)=\ln \left(k_{\mathrm{w}}\right)-S \cdot \mathrm{c}$.

where $k_{\mathrm{w}}$ is the extrapolated value of $k$ for $<!_{0}$, i.e., in pure water and $S$ is the solvent strength parameter, which is a 

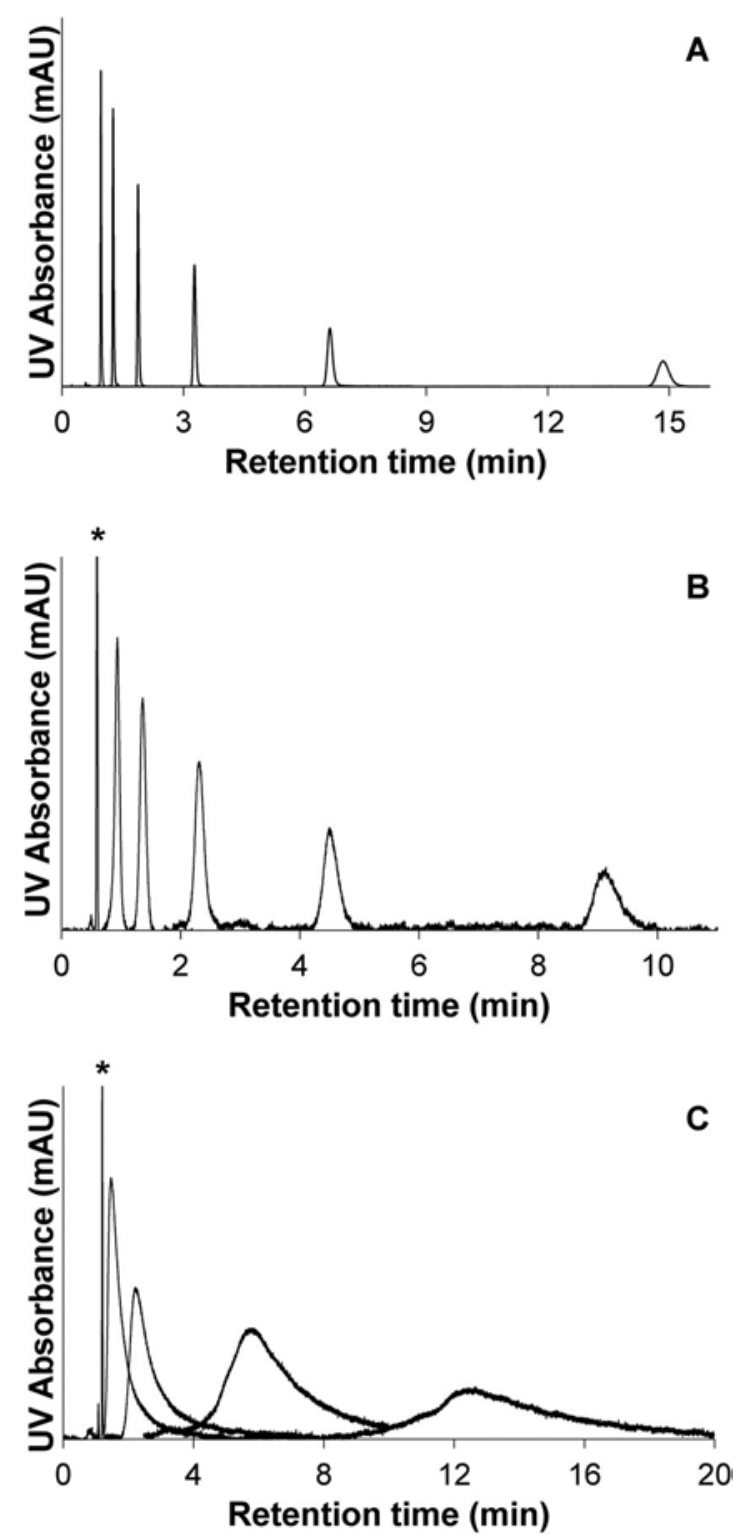

Figure 1. Overlay of the peak profiles measured in isocratic mode for (A) 2-nitrophenol employing 60 (first-eluting peak), 50, 40, 30, 20 , and $10 \%$ ACN (last-eluting peak) as mobile phase; (B) insulin employing 28 (first-eluting peak), 27, 26, 25, and 24\% ACN (lasteluting peak) as mobile phase; and (C) cytochrome c employing 25.6 (first-eluting peak), 25.2, 24.8, 24.4, and 24\% ACN (last-eluting peak) as mobile phase. The asterisk marks the injection pulse. The small molecule and peptide separations were obtained on a $\mathrm{C}_{18}$ column, proteins on $\mathrm{a}_{4}$ column. The applied flow rate was $0.3 \mathrm{~mL} / \mathrm{min}$.

constant for a given analyte and organic solvent. The LSS model has been applied for both small-molecule separations [12-14] and biomolecules, including peptides and proteins [15-17]. However, due to strong dependency of the retention time of biomolecules on solvent composition, almost no isocratic data are readily available to establish and validate retention-time models. Furthermore, it has been reported that the LSS model (for small-molecule separations) is only valid in a narrow range of <! values [18]. To account for this aspect, and also to improve retention-time modeling when multimodal retention mechanisms occur, nonlinear retention-time models have been proposed in literature, including the quadratic model (Eq. 2) [8] and the model developed by Neue and Kuss (Eq. 3) [11]:

$\ln (k)=\ln \left(k_{\mathrm{w}}\right)-S_{1} \cdot$ c. $-S_{2} \cdot$ c. $^{2}$

$\ln (k)=\ln \left(k_{\mathrm{w}}\right)+2 \cdot \ln \left(1+S_{2} \cdot \mathrm{c}.\right)-\frac{S_{1} \cdot \mathrm{c} .}{1+S_{2} \cdot \mathrm{c} .}$

where $k_{\mathrm{w}}$ is the extrapolated intercept, $S_{1}$ is the slope, and $S_{2}$ is the curvature coefficient. The Neue model also shows a strong resemblance to the equation used by Bosch and Rosés [19], using the polarity index as a measure of elution strength of a mobile phase, which extends the applicability of the model over the full range of mobile-phase compositions. The models defined by Eqs. (2) and (3) can accurately fit the concave trend that may become apparent when using acetonitrile instead of methanol as an organic modifier, or when undesired secondary retention effects with silanol groups present at the stationary phase surface affect retention [20, 21]. However, the main disadvantage of these models is that a large number of isocratic data points is required to accurately fit the curvature between $\ln k$ and <! [22]. Furthermore, the quadratic model is likely to provide an erroneous value of $k_{\mathrm{w}}$ during extrapolation [11].

In this study, we investigated the possibilities and limitations of the applicability of the LSS model, the quadratic retention-time model, and the Neue retention-time model to accurately estimate gradient retention factors for small molecules, i.e., phenol derivatives, and biomolecules including synthetic peptides and intact proteins with molecular weight (MW) values ranging between 817 and 66430 Da. Retention-model parameters have been based on isocratic retention-time RP-LC measurements applying mobile phases with different solvent strength. The accuracy of retentiontime predictions based on LSS $k_{\mathrm{w}}$ and $S$ parameters and the $k_{\mathrm{w}}, S_{1}$, and the $S_{2}$ for the nonlinear models have also been assessed by performing scouting runs in gradient mode applying different combinations of gradient times and gradient windows.

\section{Material and methods}

\subsection{Chemical and materials}

Acetonitrile (ACN, HPLC supragradient quality) and formic acid (FA) were obtained from Biosolve (Valkenswaard, The Netherlands). Deionized HPLC-grade water was produced in-house using a Milli-Q water purification system (Millipore, Molsheim, France). Uracil, 5-amino-2nitrophenol (1), 4-nitrophenol (2), 3-nitrophenol (3), o-cresol (4), 2-nitrophenol (5), 4-bromphenol (6), 3,4-dimethylphenol 

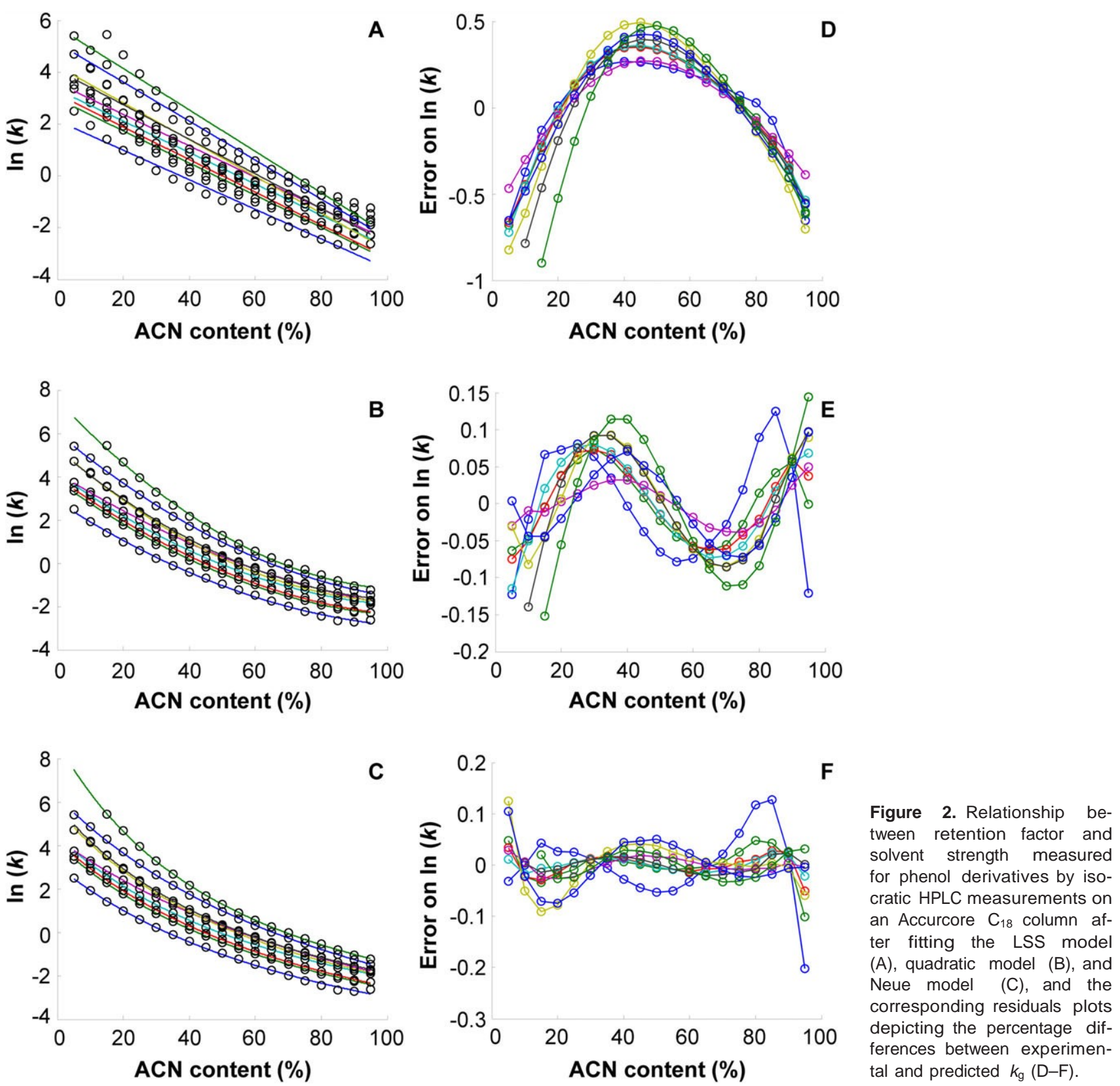

(7), 4-bromo-2-nitrophenol (8), 4-bromo-2,6-xylenol (9), five synthetic peptides, i.e., neurotensin (10), angiotensin I human acetate salt hydrate (11), bradykinin fragment 1-8 acetate salt hydrate (12), adrenocorticotropic hormone fragment 18-39 human (ACTH; 13), and insulin chain B oxidized from bovine pancreas (14), and three proteins, i.e., cytochrome c from equine heart (15), carbonic anhydrase from bovine erythrocytes (16), and BSA (17) were purchased from Sigma-Aldrich (Steinheim, Germany).

A stock solution of uracil and individual phenol derivatives was prepared by dissolving $500 \mathrm{ppm}$ of each compound in 70:30\% v/v ACN/water. For each peptide and protein, a stock solution was prepared by dissolving $1000 \mathrm{ppm}$ of each analyte in water containing $0.05 \% \mathrm{v} / \mathrm{v}$ FA. The individual phenol, peptide, and protein sample mixtures were prepared by diluting the stock solutions to concentrations in the range of 40-80 ppm for phenols, 4-100 ppm (bradykinin and neurotensin), 10-100 ppm (insulin and angiotensin), 20$200 \mathrm{ppm}$ (ACTH), and 100-1000 ppm (cytochrome c, carbonic anhydrase, and BSA). All samples were dissolved in mobile-phase compositions corresponding to either the isocratic elution conditions or gradient starting conditions.

For the separation of phenols and peptides an Accucore $\mathrm{C}_{18}$ column $(100 \times 2.1 \mathrm{~mm}$ id, pore size: $80 \AA)$ packed with 2.6 f.Lm core-shell $\mathrm{C}_{18}$ silica-modified particles obtained from 

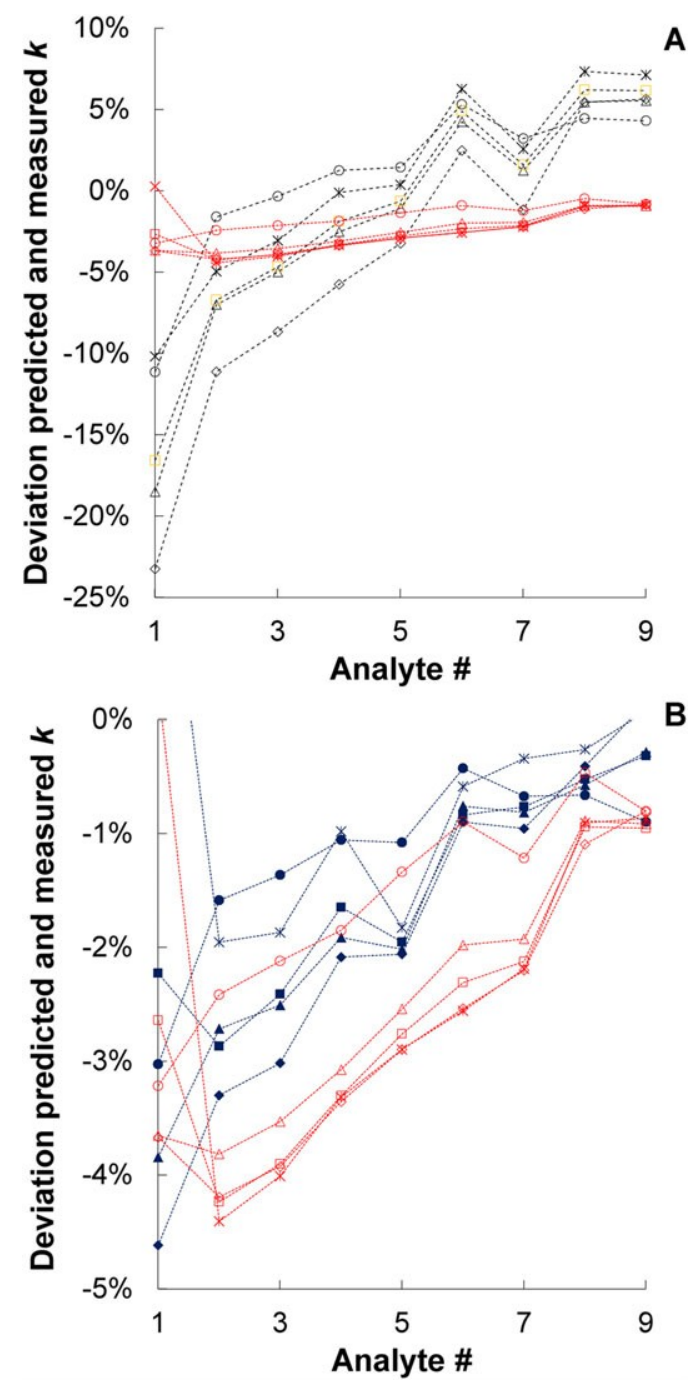

Figure 3. Deviation in predicted gradient retention factor based on $S$ and $k_{w}$ values determined using isocratic retention-time measurements and experimental retention factors measured in gradient mode. (A) Comparison of deviations in $k_{\mathrm{g}}$ based on LSS model (black striped line) and Neue model (red dotted line) and (B) Comparison of deviations in $\mathrm{k}_{\mathrm{g}}$ based on quadratic model (blue striped line) and Neue model (red dotted line). Different symbols represent different gradient conditions, i.e., 5-95\% ACN with $t_{\mathrm{G}}=5 \mathrm{~min}$ (circles); $5-95 \% \mathrm{ACN}$ with $t_{\mathrm{G}}=10 \mathrm{~min}$ (triangles), $5-95 \% \mathrm{ACN}$ with $t_{\mathrm{G}}=15 \mathrm{~min}$ (diamonds); $10-95 \% \mathrm{ACN}$ with $t_{\mathrm{G}}=$ $10 \mathrm{~min}$ (squares), $15-95 \% \mathrm{ACN}$ with $t_{\mathrm{G}}=10 \mathrm{~min}$ (stars).

Thermo Fisher Scientific (Germering, Germany) was used. Protein separations were conducted using an Accucore-150$\mathrm{C}_{4}$ column $(150 \times 2.1 \mathrm{~mm}$ id, pore size: $150 \AA)$ packed with 2.6f.Lm core-shell $\mathrm{C}_{4}$ silica-modified particles (Thermo Fisher Scientific).

\subsection{Instrumentation and LC conditions}

HPLC experiments were conducted using an Ultimate 3000 RSLC system (Thermo Fisher Scientific) composed

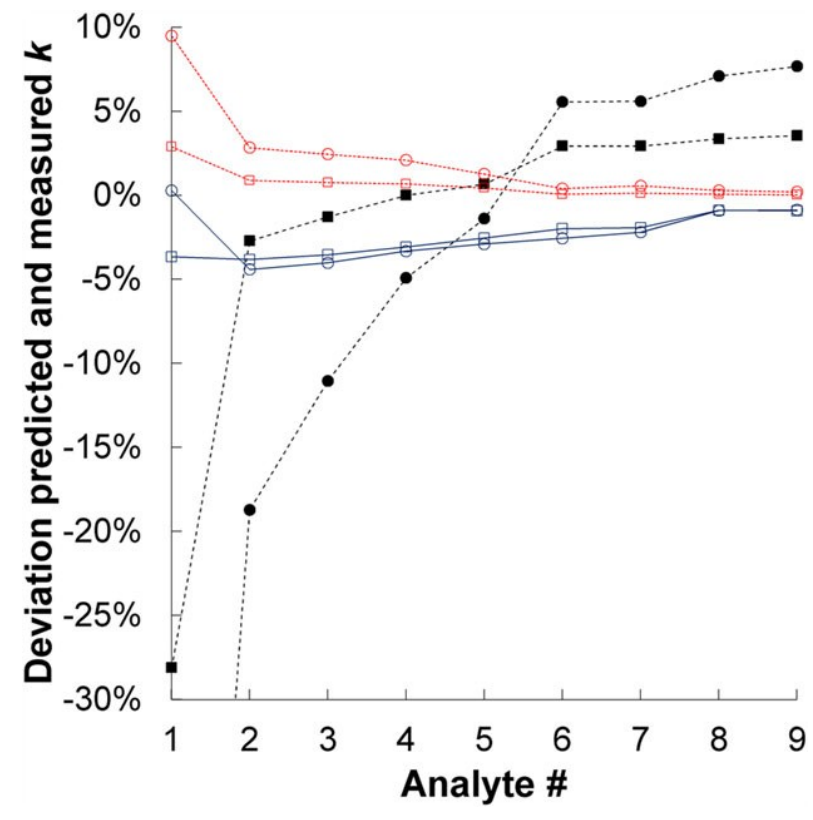

Figure 4. Comparison of the retention-time prediction accuracy based on LSS model (closed symbols) and Neue model (open symbols) of individual nitrophenols. Neue model built on $<!=$ $0.05-0.95$ with $t_{\mathrm{G}}=5,10$, and $15 \mathrm{~min}$ (red dotted lines); Neue model built on $<!=0.05-0.95$ with $t_{G}=5$ and $10 \mathrm{~min}$, and $<!=$ $0.10-0.95$ with $t_{G}=10 \mathrm{~min}$ (blue full lines).

of degasser, a binary high-pressure pump, a thermostatted pulled-loop autosampler equipped with a six-port injection valve containing a 1.2 f.LL injection loop, a temperaturecontrolled column compartment set at $30^{\circ} \mathrm{C}$, and a diode array detector with a 2.5 f.LL flow cell. NanoViper tubing (Thermo Fisher Scientific) was used to connect the column with injector and detector (inlet and outlet tubing $350 \times 0.075 \mathrm{~mm}$ id).

The external time $\left(t_{\text {ext }}\right)$ and pressure contributions induced by connection tubing were determined by injecting uracil while replacing the column with a zero-dead volume connector. The column dead time $\left(t_{0}\right)$ was determined by injecting uracil applying a mobile-phase composition of 76:24\% $\mathrm{v} / \mathrm{v}$ of ACN/water. The column dead time corrected for external contributions was $0.54 \mathrm{~min}$. The dwell time $\left(t_{\mathrm{D}}\right)$ was determined by adding acetone tracer in the mobile phase, recording a blank gradient run, and performing UV detection at $254 \mathrm{~nm}$. $t_{\mathrm{D}}$ was determined to be $1.4 \mathrm{~min}$ at $0.3 \mathrm{~mL} / \mathrm{min}$.

Phenols were separated under isocratic and gradient conditions using water and ACN as mobile phase. The isocratic separations of phenol derivatives were measured in a wide range of acetonitrile/water ratios, with $<$ ! ranging between 0.05 and 0.95 ACN. A mixture containing nine phenols and uracil was separated applying linear gradients with initial mobile-phase compositions $\left(<!_{0}\right)$ starting at $0.05,0.10$, and 0.15 ACN to a final mobile-phase composition ( $<$ !end) containing 0.95 ACN. Gradient separations were conducted applying gradient times $\left(t_{\mathrm{G}}\right)$ of 5,10 , and $15 \mathrm{~min}$, respectively. Peptides and proteins were separated under isocratic and gradient 

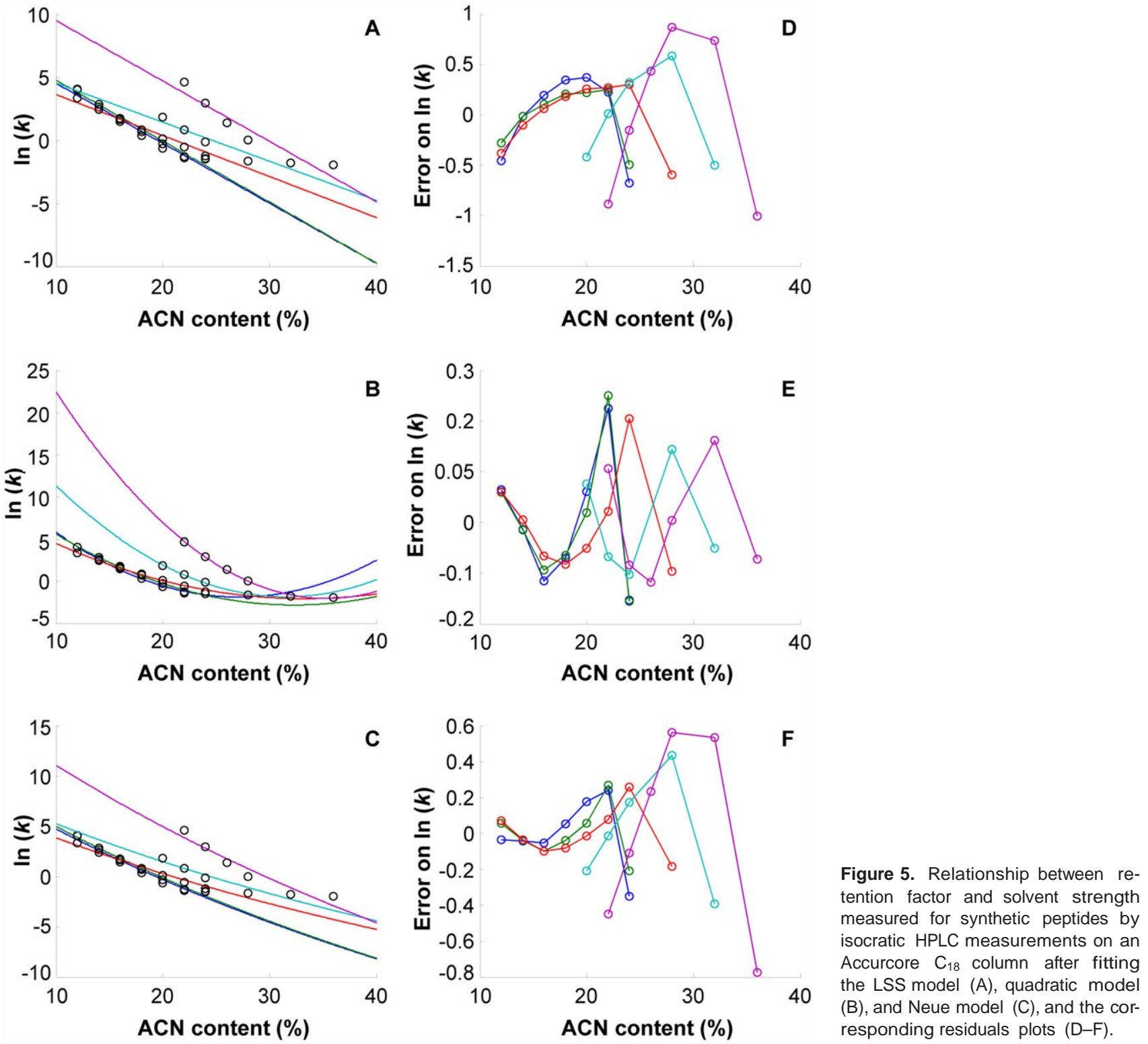

conditions using aqueous ACN mixtures containing 0.05\% v/v FA. Linear gradient separations were conducted applying $<!_{0}$ values of $0.04,0.08,0.12,0.16$, and 0.20 and $<!$ end 0.64 ACN, with $t_{\mathrm{G}}$ of $3.42,5,10$, and $15 \mathrm{~min}$. All HPLC experiments were performed applying a flow rate of $0.3 \mathrm{~mL} / \mathrm{min}$, corresponding to the optimal van-Deemter flow rate for smallmolecule separations.

\subsection{Numerical methods}

To obtain the $k_{\mathrm{w}}$ and $S$ parameters from isocratic retentiontime measurements, an in-house written Matlab program that employs least-square fitting was used. For the nonlinear models, fitting was performed by the Matlab routine lsqcurvefit. A grid search in starting values was implemented. For the

linear and quadratic model, using isocratic runs, the Matlab routine polyfit was used. For the linear model, using gradient scouting runs, the analytical expression for the gradient retention factor was implemented in the Matlab routine lsqcurvefit. The residuals of the isocratic fitting were calculated as $\left(k_{\text {predicted }}-k_{\exp }\right)$ and the percentage errors were determined as $\left(k_{\text {predicted }}-k_{\exp }\right) / k_{\exp }$.

\section{Results and discussion}

To grasp as much similarities and/or differences in retention mechanisms/predictive ability of the retention models for the separation of small molecules and biomolecules, including synthetic peptides and intact proteins, all analytes were analyzed using the same instrument setup. A 
column packed with silica core-shell $\mathrm{C}_{18}$ particles was used for the separation of small molecules, i.e., selected phenol derivatives that have been listed as priority pollutants by the

United States Environmental Protection Agency (EPA) [23].

To cover a broad hydrophobicity range, a test mixture containing nitro-, alkyl-, and bromophenol derivatives was compiled for this study. The peptides were also analyzed using the $\mathrm{C}_{18}$ column and the MW of the synthetic peptides ranged between 817 and 5734 Da. Intact proteins with MW of 12 384, 29000 , and 66430 Da were separated on a packed column with $\mathrm{C}_{4}$ functionality. Insulin, composed of 51 amino acids, bridges the gap between the small MW peptides and intact proteins.

After conducting an initial gradient scouting run to estimate the applicable range of mobile-phase compositions, isocratic retention-time measurements were conducted. Although we opted to reach the same $k$-range as for the small molecules by selection of biomolecules with a broad MW range, the applicable solvent composition range for peptides and especially proteins was much smaller compared to the small molecules. Figure 1 shows an overlay of elution profiles of a selected phenol (Fig. 1A), a peptide (Fig. 1B), and an intact protein (Fig. 1C) obtained in isocratic mode at different $<$ ! values. With increasing MW the applicable <! range decreases, corresponding to a higher solvent strength factor $S$. Indeed, a relationship between the $S$-value and the MW of the molecules was already proposed by Snyder and Dolan [7]. Using low solvent strength, broad peaks and a low $\mathrm{S} / \mathrm{N}$ are obtained affecting the accurate determination of the retention time. To account for dispersion, limiting the detectability, the concentration of the intact proteins was increased.

\subsection{Retention-time modeling of phenol derivatives}

Isocratic retention-time measurements were performed and the relationship between retention factor $(k)$ and solvent strength (<!) was modeled using the LSS model (Fig. 2A), the quadratic retention time-model (Fig. 2B), and the Neue model (Fig. 2C). Figure 2D and E show the corresponding residual plots. The nonlinear retention behavior of the phenol derivatives is most pronounced at low and high ACN content, and is only taken into account by the quadratic and Neue model. Although an S-shaped trend in residuals is observed when applying the quadratic retention-time model, the absolute values in residuals for the nonlinear retentiontime models are in both cases relatively small. Next, $k_{\mathrm{g}}$ can be estimated using the following analytical expressions for the LSS [24] and the Neue model, respectively [11]:

$$
k=\frac{t_{\mathrm{D}}}{t_{0}}+\frac{1}{S \cdot \frac{\mathrm{c} .}{t_{\mathrm{G}}} \cdot t_{0}} \cdot \ln \quad t_{0}-\frac{t_{\mathrm{D}}}{k} \underbrace{\backslash}_{0} \cdot \frac{S \cdot \frac{\mathrm{c} .}{t_{\mathrm{G}}} \cdot t_{0} \cdot k_{0}}{t}+1
$$

$$
\begin{aligned}
& k_{\mathrm{g}}=\frac{t_{\mathrm{D}}}{t_{0}}
\end{aligned}
$$

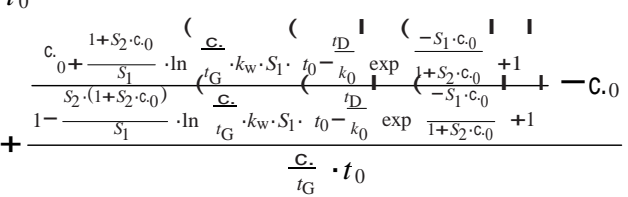

where $k_{0}$ represents the analyte retention factor at the start of the gradient. Determining $k_{\mathrm{g}}$ for the quadratic model involved numerical integration [25]. The experimental gradient retention factors $\left(k_{\mathrm{g}}=\frac{t_{\mathrm{R}}}{t_{0}}-1\right)$ were determined for all individual analytes directly from the chromatograms recorded in gradient mode while applying $t_{\mathrm{G}}=5,10$, and 15 min with $<!_{0}-<!_{\text {e }}$ from 0.05 to 0.95 , and a fixed gradient time $\left(t_{\mathrm{G}}=\right.$ $10 \mathrm{~min})$ with different gradient starting conditions $\left(<!_{0}=0.10\right.$ and 0.15 , respectively). Figure 3 shows the deviation between the experimental $k_{\mathrm{g}}$ and $k_{\mathrm{g}}$ values based on Eqs. (4) and (5) predicted by $S$ and $k_{\mathrm{w}}$ parameters measured in isocratic mode for each analyte individually. In Fig. 3A, the prediction errors using the LSS and Neue models are compared. The largest deviation between the predicted and measured $k_{\mathrm{g}}$ value applying the LSS model is observed for early eluting analytes. The nonlinearity present in the isocratic curve is accounted for by the Neue model, which leads to more accurate predictions of $k_{\mathrm{g}}$. The quadratic model was found to perform slightly better than the Neue model (average absolute prediction error of 1.5 versus $2.4 \%$ ), see Fig. $3 \mathrm{~B}$.

The $k_{\mathrm{w}}$ and $S$ parameters for the LSS and Neue model can also be estimated by gradient scouting runs using three different $t_{\mathrm{G}}$ and two different $<!_{0}$ and applying Eqs. (4) and (5), employing a leave-two-out strategy to estimate $S\left(S_{1}\right.$ and $S_{2}$ ) and $k_{\mathrm{w}}$ values. Figure 4 compares the deviation between experimental $k_{\mathrm{g}}$ and predicted values based on LSS and Neue models for each analyte individually. The prediction accuracy using the LSS model decreases significantly for early eluting analytes in case the gradient starts at relatively high solvent strength. The predictions using the nonlinear Neue model outperform those obtained by applying the LSS model. Figure 4 also shows that the prediction accuracy depends on $t_{\mathrm{G}}$ and $<$ ! combination used during building of the model. Overall, predictions for $k_{\mathrm{g}}$ using isocratic retention-time measurements and application of the Neue model were most accurate.

\subsection{Retention-time modeling of peptides and intact proteins}

Figures 5 and 6 show the LSS, quadratic, and Neue retentiontime model fits and their residuals for peptides and intact proteins, respectively. The peptides depict a clear nonlinear retention behavior, hence the nonlinear retention-time model fits were more accurate. The residuals of the quadratic model are lower than those of the Neue model, and the Neue

model cannot cope with the strong nonlinearity that is present for the two most retained peptides (ACTH and insulin). Even 

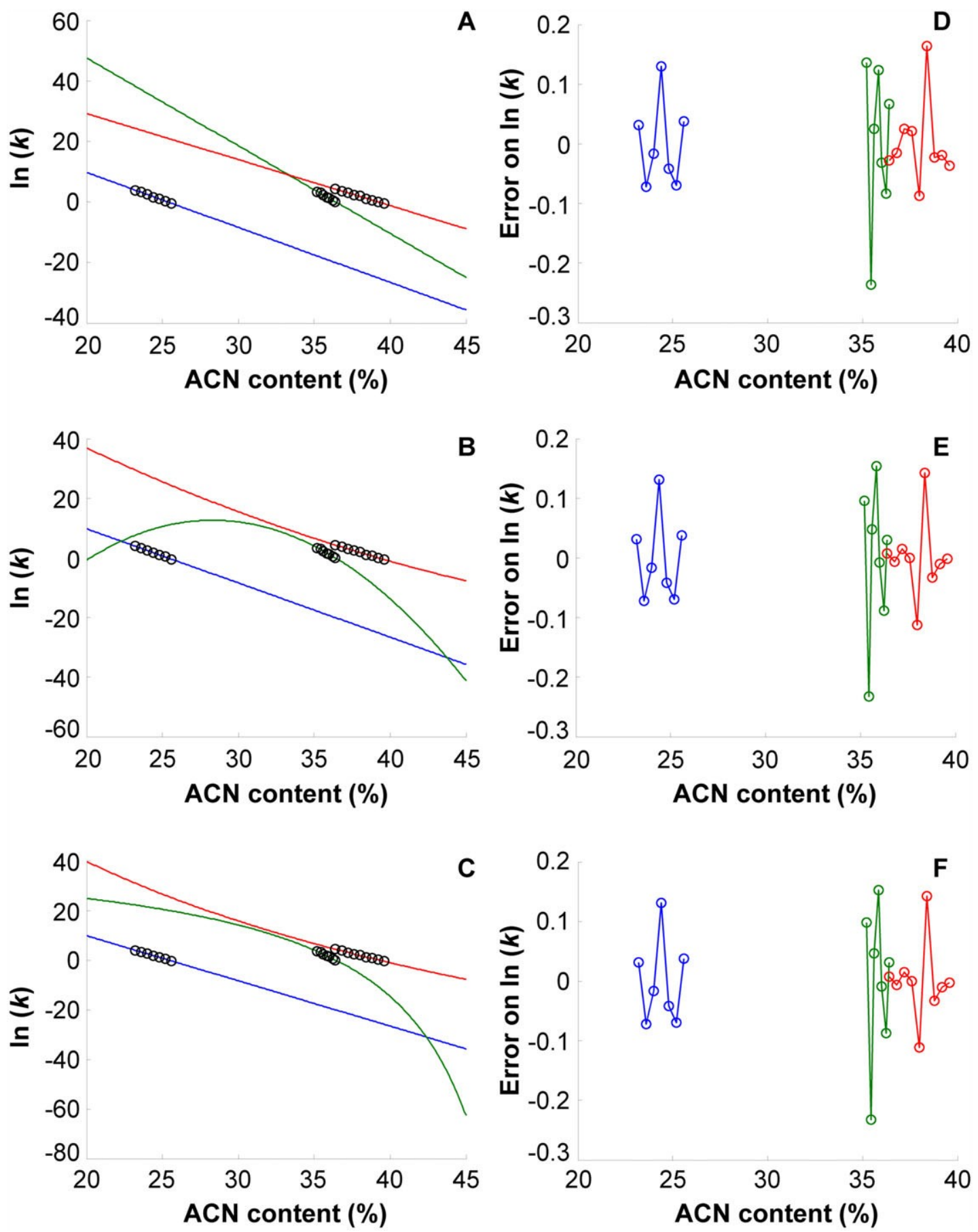

Figure 6. Relationship between retention factor and solvent strength measured for intact proteins by isocratic HPLC measurements on an Accurcore $\mathrm{C}_{18}$ column after fitting the LSS model (A), quadratic model (B), and Neue model (C), and the corresponding residuals plots $(\mathrm{D}-\mathrm{F})$.

when using a $\mathrm{C}_{4}$ column, only a very small elution window could be applied for the retention-time determination of intact proteins. This resulted in an almost linear ln $(k)$ versus <! domain. At these conditions, the LSS model accurately describes the retention behavior. At this point it can only be speculated that outside the <! range, intact proteins feature nonlinear retention behavior, similar to peptides, and that the extrapolation to determine $k_{\mathrm{w}}$ may lead to significant underestimation compared to its true value.

Using a similar strategy as applied in Section 3.1, the gradient retention factors were estimated based on isocratic model parameters and compared to experimental values recorded in gradient mode. Figure 7A compares the LSS, Neue, and quadratic model predictions for a gradient time of 

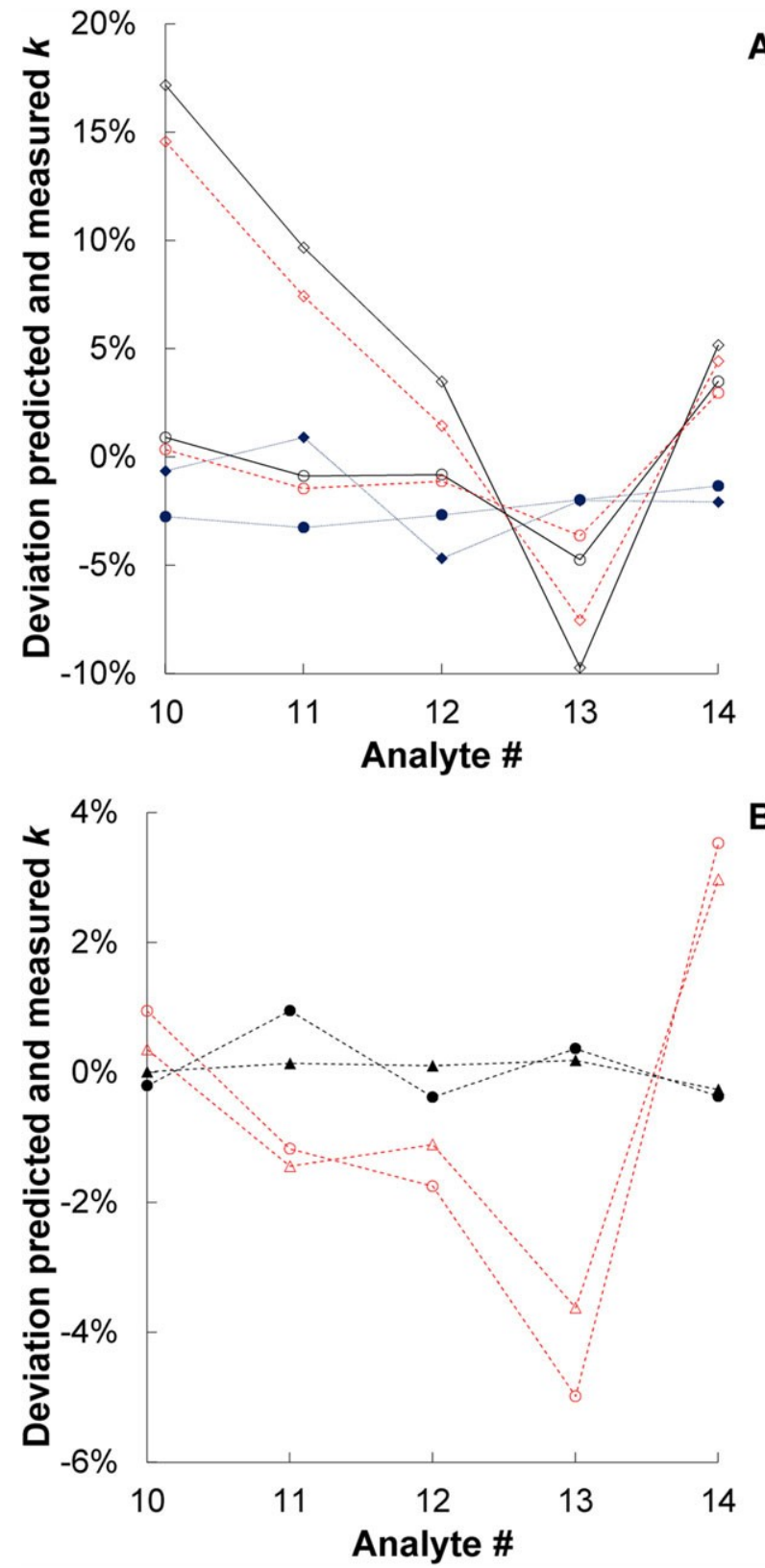

Figure 7. Comparison of the retention-time prediction accuracy for peptides, based on isocratic measurements and applying the LSS (black solid line), quadratic (blue dotted line), and Neue models (red striped line) (A), and comparison of prediction accuracy based on isocratic data (red open symbols) and gradient scouting runs (black solid symbols) using the Neue model. Gradient conditions: $<!=0.08-0.64$ with $t_{G}=15 \mathrm{~min}$ (circles); $<!=0.12-$ 0.64 with $t_{G}=15 \mathrm{~min}$ (triangles); $<!=0.15-0.64$ with $t_{G}=15 \mathrm{~min}$ (diamonds).

15 min applying a broad gradient span $(<!=0.04-0.64)$ and a gradient starting at higher ACN content $(<!=0.20-0.64)$, respectively. Although the LSS fit is poor, the prediction accuracy for peptides applying the LSS model is surprisingly good when applying a broad gradient span (starting at low ACN content). However, when using a relatively high con- tent of ACN at the start of the gradient, high prediction errors in the range up to $17 \%$ are found for the first eluting peptide. The Neue model provides more accurate $k_{\mathrm{g}}$ predictions, but also started to deviate significantly when high content of the organic modifier is used during the gradient start. The quadratic model yielded the best results, with only an average prediction error of $2.3 \%$. The use of gradient scouting runs in combination with the Neue model and the analytical expression in Eq. (5) resulted in better prediction errors compared to the use of isocratic runs, see Fig. 7B. The prediction error ranged typically between 0.1 and $1.3 \%$ and the average prediction error was $2.2 \%$. Only when the organic modifier content at the gradient start was increased $\left(<!_{0}>0.16\right)$, the accuracy for the early eluting peptides decreased, which may be caused by partial isocratic elution behavior. The use of gradient scouting runs has the additional advantage of being faster and more generic in nature. Note that a grid search in the starting values of the retention parameters was needed during the least-squares optimization to find an acceptable fitting.

As shown in Fig. 6, an almost linear relationship was obtained between $\ln k$ and $<$ in the solvent strength window that could be practically applied. Hence, for the intact proteins, the $S$ and $k_{\mathrm{w}}$ parameters were only determined applying the LSS model. Based on isocratic retention-time measurements the average prediction error was $3.2 \%$ with a maximum error of $4.8 \%$ (obtained when applying $<!_{0}=0.16$ and $t_{\mathrm{G}}=15 \mathrm{~min}$ ). Using a CAMD strategy, we also investigated the use of gradient scouting runs to build the LSS and Neue retention-time models. However, no acceptable fitting could be obtained, even with the use of a grid search with initial values for the retention parameters, and by changing the solver options to make the optimization as sensitive as possible. We also considered different combinations of scouting runs (with different gradient starting concentrations), but these resulted in an improper fit.

\section{Concluding remarks}

For both the small molecules and the peptides, the attained isocratic $k$-values correspond to a relatively large solvent range in which nonlinearity is present. Hence, a better description of the isocratic retention-time measurements can be found using the nonlinear retention models. However, for the most retentive peptides studied (ACTH and insulin) the error on prediction accuracy increased significantly using the Neue model. For both the small-molecule and peptide separations, the quadratic model was found to provide more accurate retention time predictions than the Neue model. Considering the fact that an analytical expression for $k_{\mathrm{g}}$ exists for the Neue model, allowing fast and generic scouting runs, the Neue model can be seen as the preferred model for gradient prediction of small molecules and peptides. Moreover, the prediction errors were reduced using the Neue model in case of peptide gradient separations. The resulting prediction errors 
were in a similar range to those obtained by the quadratic model and isocratic scouting runs.

In agreement with the higher MW of the proteins and the expected high $S$ values [7], the ln $(k)$ versus <! curve was found to be very steep, resulting in only a very small solvent range that can be used to achieve reliable peak integration (while obtaining a similar $k$-range compared to the small molecules and peptides). The LSS model and the considered nonlinear models gave very similar fitting accuracy within the solvent strength range applied. Outside of this range, the nonlinear models yielded physically impossible extrapolations. Although it can be speculated that proteins should exhibit nonlinear retention behavior, similar to peptides, the solvent range that can be practically applied is too small to demonstrate this. The use of gradient scouting runs to accurately estimate the retention parameters in case of protein separation was unsuccessful. Hence, for CAMD of proteins, individual isocratic runs (at least two) should be performed to obtain the retention parameters.

This work was supported by a grant from the Research Foundation Flanders (G.0256.16N). N. V. acknowledges a grant from the Erasmus+ program.

The authors have declared no conflict of interest.

\section{References}

[1] Nikitas, P., Pappa-Louisi, A., Agrafiotou, P., J. Chromatogr. A 2004, 1034, 41-54.

[2] Snyder, L. R., Dolan, J. W., The Practical Application of the Linear Solvent Strength Model, Wiley Interscience, Hoboken 2007.

[3] Duarte, R. M. B. O., Duarte, A. C., J. Chromatogr. A 2010, 1217, 7556-7563.

[4] Davis, J. M., Giddings J. C., Anal. Chem. 1985, 57, 21682177.

[5] Beinert, W.-D., Eckert, V., Galushko, S., Tanchuk, V., Shishkina, I., LC-GC Eur. Online Suppl. 2001, 34-38.
[6] Molnar, I., J. Chromatogr. A 2002, 965, 175-194.

[7] Snyder, L. R., Dolan, J. W., Adv. Chromatogr. 1998, 38, 115-187.

[8] Schoenmakers, P. J., Billiet, H. A. H., Tijssen, R., De Galan, L., J. Chromatogr. 1978, 149, 519-537.

[9] Neue, U. D., Mendez, A., J. Sep. Sci. 2007, 30, 949-963.

[10] Baeza-Baeza, J. J., Carcia-Alvarez-Coquie, M. C., J. Sep. Sci. 2014, 37, 2269-2277.

[11] Neue, U. D., Kuss, H.-J., J. Chromatogr. A 2010, 1217, 3794-3803.

[12] Kaliszan, R., Baczek, T., Bucinski, A., Buszewski, B., Sztupecka, M., J. Sep. Sci. 2003, 26, 271-282.

[13] Tate, P. A., Dorsey, J. G., J. Chromatogr. A 2004, 1042, 37-48.

[14] Baczek, T., Kaliszan, R., J. Chromatogr. A 2002, 962, 4155.

[15] Vankova, N., De Vos, J., Tyteca, E., Desmet, G., Edge, T., Ceslova, L., Cesla, P., Eeltink, S., J. Chromatogr. A. 2015, 1409, 152-158.

[16] Vaast, A., Tyteca, E., Desmet, G., Schoenmakers, P. J., Eeltink, J. Chromatogr. A 2014, 1355, 149-157.

[17] Hahn R., J. Sep. Sci. 2012, 22, 3001-3032.

[18] Jandera, P., Kubat J., J. Chromatogr. A 1990, 50, 281-299.

[19] Vivó-Truyols, G., Torres-Lapasió, J. R., García-AlvarezCoque, M. C., J. Chromatogr. A 2003, 1018, 169-181.

[20] Valko, K., Snyder, L. R., Glajch, J. L., J. Chromatogr. A 1993, 656, 501-520.

[21] Baczek, T., Markuszewski, M., Kaliszan, R., van Straten, M. A., Claessens, H. A., J. High Resolut. Chromatogr. 2000, 23, 667-676.

[22] Jandera, P., Hájek, T., Vynuchalová, K., J. Chromatogr. A 2014, 1337, 57-66.

[23] Michałowicz, J., Duda, W., Pol. J. Environ. Stud. 2007, 16, 347-362.

[24] Quarry, M. A., Grob, R. L., Snyder, L. R., Anal. Chem. 1986, 58, 907-917.

[25] Concha-Herrera, V., Vivó-Truyols, G., Torres-Lapasió, J. R., García-Alvarez-Coque, M. C., J. Chromatogr. A 2005, 1063, 79-88. 